\title{
Synthesis of virtual Brillouin frequency shift profiles in BOTDA sensors using optical source dithering
}

\author{
Javier Urricelqui, Mikel Sagues, Alayn Loayssa* \\ Universidad Pública de Navarra, Campus Arrosadia 31006, Spain
}

\begin{abstract}
We demonstrate a novel concept for Brillouin optical time domain analysis sensors that is based on synthesizing a virtual Brillouin frequency shift profile along the sensing fiber. The technique is based on modulating the wavelength of the optical source with a periodic waveform that is synchronized to the pump pulse. Application of this new tool to the compensation of non-local effects and Brillouin-induced noise in distributed sensors is experimentally demonstrated.
\end{abstract}

Keywords: Brillouin optical time domain analysis, Brillouin distributed sensors, non-local effects, Brillouin threshold.

\section{INTRODUCTION}

Brillouin optical time domain analysis (BOTDA) sensors are bound to become a fundamental tool for the monitoring of large structures such as pipelines or high voltage electric lines through distributed measurements of temperature or strain. However, several effects constrain the ultimate distance range of BOTDA sensors. One of the main limitation arises from non-local effects, which restrict the maximum power of the probe wave and hence the received signal-to-noise ratio (SNR). They are due to the transfer of power between the pump and probe waves: as the pump propagates along the fiber it gives energy to the probe and hence is depleted. This attenuation has a wavelength dependency that introduces an additional transfer function to the measured Brillouin spectra, which leads to measurement errors ${ }^{1}$. Several methods have been demonstrated to counteract this limitation by using special configurations of the BOTDA sensor, such as the use of a double probe wave $^{1}$ or our proposal for using an RF phase modulated probe wave ${ }^{2}$. Another factor that limits the injected probe power is noise initiated from spontaneous Brillouin scattering (SpBS), which leads to probe depletion and noise in the detected signal ${ }^{3}$. This is another manifestation of the so-called Brillouin threshold of a fiber link.

In this work, we introduce a novel tool to extend the reach of BOTDA sensors that is based on synthesizing a virtual Brillouin frequency shift (BFS) profile along the sensing fiber by modulating the wavelength of the optical source. We demonstrate application of this virtual BFS to simultaneously compensate non-local effects and Brillouin noise.

\section{FUNDAMENTALS OF THE METHOD}

The fundamentals of the technique that we propose are schematically depicted in Fig. 1. This figure describes a conventional BOTDA setup based on a single source, although the technique is also applicable to double laser setups. The laser source is divided in two branches. As it is well-known, a basic BOTDA setup requires the generation of a pump pulse and a CW probe that have a controlled wavelength difference, which should be tunable around the Brillouin frequency shift (BFS) of the deployed fiber. In Fig. 1, the upper branch is used to generate the pump pulses by pulsing the laser source signal and the lower branch is used to generate the probe wave. Depending on the particular type of BOTDA setup deployed, the frequency translation of the laser source signal required to have the tunable wavelength difference between pump and probe that is needed for Brillouin interaction may be performed either in the upper or lower branch, typically by the sideband generation technique, i.e. using modulation in the microwave range to generate sidebands of the narrow laser source.

The only modification to a conventional BOTDA in this setup is that the laser wavelength is modulated by a low-frequency periodic wave that is synchronized to the pulse generator. This dithering is performed using small current modulation by taking advantage of semiconductor laser's adiabatic chirp. A sinusoidal wave is depicted in the figure as an example, but any other modulation shapes are possible. Furthermore, the modulation shape is directly translated to the synthesized virtual BFS profile along the fiber as it is explained below.

*alayn.loayssa@unavarra.es; phone 0034 948169840; fax 0034 948169720; unavarra.es 

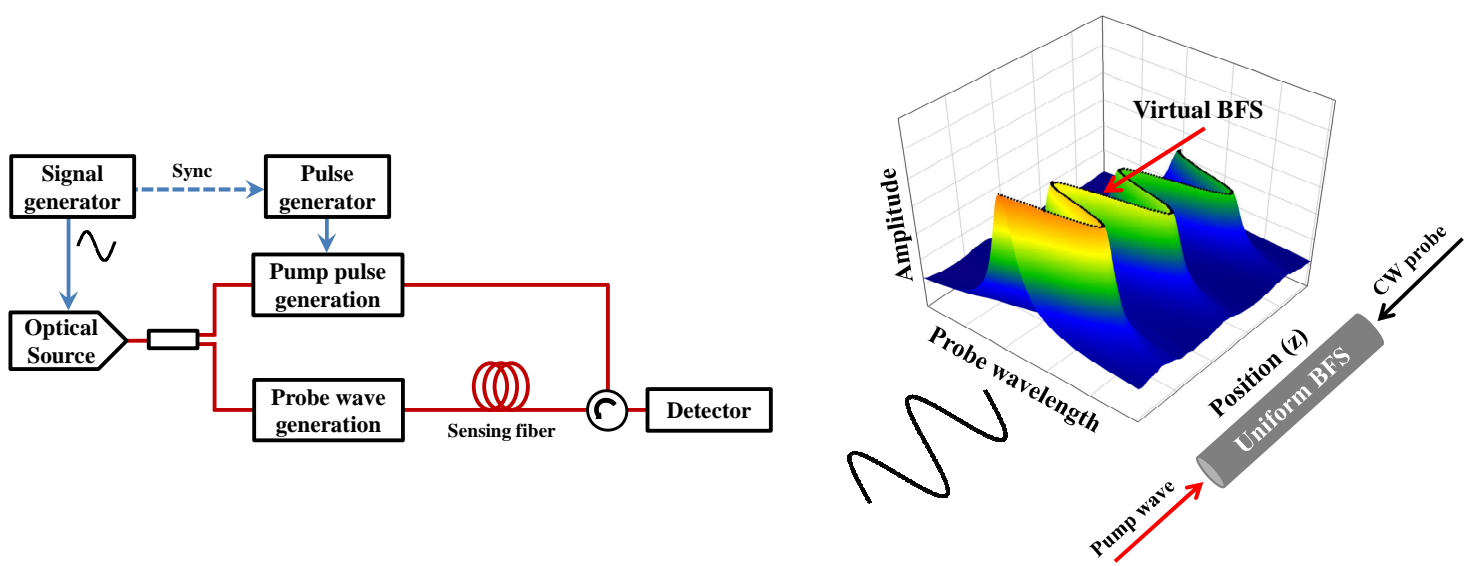

Figure 1. Fundamentals of the laser wavelength dithering technique for virtual BFS synthesis.

In the upper branch the wavelength modulated source is pulsed and, as the pulse generator and wavelength modulation are synchronized, the pump pulse acquires a wavelength that depends on the fixed propagation delay to the pulse modulator. Then, the pump pulse counter-propagates with the probe wave. However, in contrast to conventional BOTDAs, the probe wave wavelength is not constant along the fiber because the laser source is modulated. Therefore, at each location in the fiber, $z$, the pump pulse meets a probe wave with a different wavelength. The Brillouin interaction between these two waves at each location is defined by the detuning from the center of the Brillouin spectrum $\Delta v(z)=v_{\mathrm{P}}-v_{\mathrm{S}}(z)-\operatorname{BFS}(z)$, where $v_{\mathrm{P}}$ is the pump pulse wavelength and $v_{\mathrm{S}}$ is the local probe wavelength. Note that expression tells us that, from the point of view of the Brillouin interaction, it is equivalent to have an actual variation in BFS due to the fiber characteristics than to have that same variation in $v_{\mathrm{S}}$. Therefore, a virtual BFS profile is synthesized by the probe wavelength modulation.

This synthesized virtual BFS profile can be used, for instance, to compensate non-local effects and Brillouin noise. Application of the synthesized virtual BFS profile to compensate non-local effects relies on the same idea that methods that use a sensing fiber made of fiber segments with different characteristic BFS ${ }^{4}$. The advantage is that we do not need to modify the fiber type along the sensing route, which is cumbersome. By using source wavelength modulation we are creating a virtual BFS profile along the sensing fiber. Non-local effects are due to the transfer of energy between the probe wave and the pump pulse. The worst-case scenario in a conventional BOTDA is when we have a fiber with uniform BFS. In this case, there is significant Brillouin interaction between pump and probe waves along the whole fiber, the energy transfer is maximum and the measurement error at the fiber's end is large ${ }^{1}$. Using our technique we can ameliorate this problem because, even though the BFS along the fiber is constant, the changing wavelength difference of pump and probe waves varies along the sensing fiber effectively synthesizing a virtual BFS. As a consequence of this position dependency, the energy transfer principally occurs at those locations, where this difference coincides with the BFS. Therefore, there is less depletion, non-local effects and measurement error.

The other effect of deploying our technique is the reduction of the Brillouin induced noise in the detected probe power. The power of the probe wave that can be injected in the fiber is limited because for high power the probe wave can pump SBS amplification of thermally-induced SpBS waves, in a process that leads to the onset of the so-called Brillouin threshold. This threshold refers to the maximum power that can be injected in a fiber before significant power begins to be reflected back and the launched signal is depleted. Moreover, as the injected probe power approaches the threshold, significant noise is added to the detected signal ${ }^{3}$ compromising SNR performance. The Brillouin threshold can be increased and the Brillouin-induced detection noise reduced by implementing the BFS synthesis technique. Again, the idea is to mimic those approaches that seek to increase the SBS threshold by using fiber links with a variation of BFS along their length ${ }^{5}$, but synthesizing a virtual SBS profile instead of implementing a real one.

\section{EXPERIMENTAL RESULTS}

The BOTDA setup that we used to demonstrate the technique is based on a phase-modulated probe wave and RF demodulation $^{2}$. However, as it was explained before, the technique is equally valid for any BOTDA setup. Figure 2 sketches the deployed experimental setup. As indicated, the setup was modified to add a function generator, which is synchronized to the pulse generator, to directly modulate the optical source, which was a standard 1560-nm high-power 
DFB laser. Sinusoidal modulation of frequency $60 \mathrm{kHz}$ and amplitude of $200 \mathrm{mV}$ was used in this proof-of-concept demonstration. The light from this modulated wavelength laser is coupled into two optical branches. In the upper branch, a double-sideband suppressed-carrier modulation is deployed with a Mach-Zehnder electro-optic modulator (MZ-EOM) and then is pulsed using a semiconductor optical amplifier (SOA). The 10-ns-temporal switching provides a pulsed signal with an acquired wavelength given by the time delay between the DFB and the SOA switch. This signal is amplified by an erbium-doped fiber amplifier (EDFA) and the upper-sideband of the pulsed signal is filtered by a narrowband fiber Bragg grating (FBG). Then, a polarization scrambler (PS) is used before launching the pulses into the sensing fiber via a circulator. In the lower branch, a probe wave is generated with an electro-optic phase modulator driven by a 1.3-GHz RF signal. Once the probe wave has interacted with the pump pulse via SBS it is photodetected and the resultant RF signal is demodulated and captured in a digital oscilloscope.

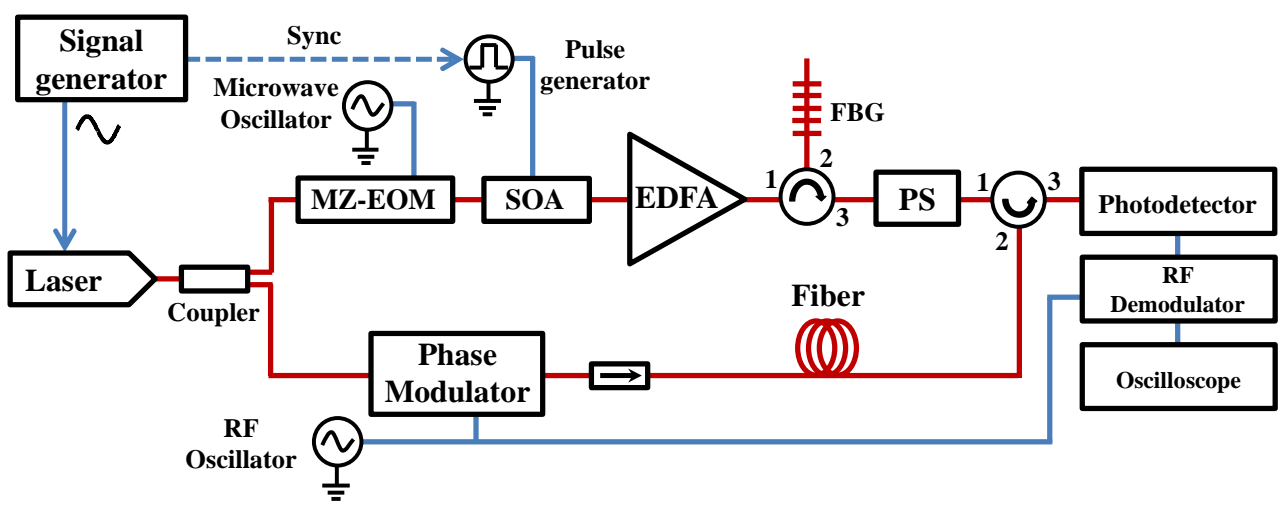

Figure 2. Experimental setup for the BOTDA sensor based on the laser wavelength dithering technique.

Fig. 3 compares the distribution of Brillouin spectra along the fiber with and without applying the wavelength modulation to the optical source. Notice that with modulation, a sinusoidal virtual BFS distribution that mimics the wavelength modulation is synthesized. A total of 12 periods of the sinusoid were distributed along the fiber. Other spectral profiles could be easily implemented, for instance, a wavelength modulation using a square wave with $5-\mathrm{kHz}$ frequency would lead to a BFS profile equivalent to that of two concatenated 10-km fiber segments with different characteristic BFS value. Note that direct modulation of the laser also generates a small sinusoidal variation of the amplitude of the measured Brillouin spectra along the fiber that has no implications on the sensor performance.

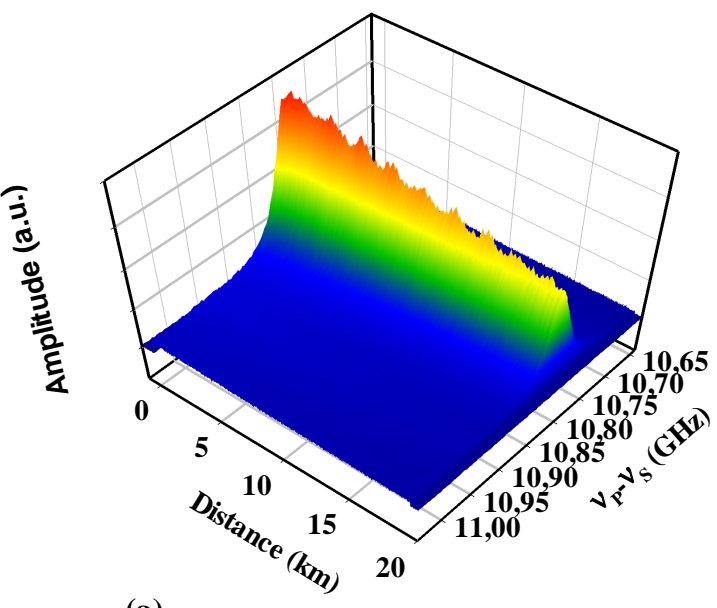

(a)

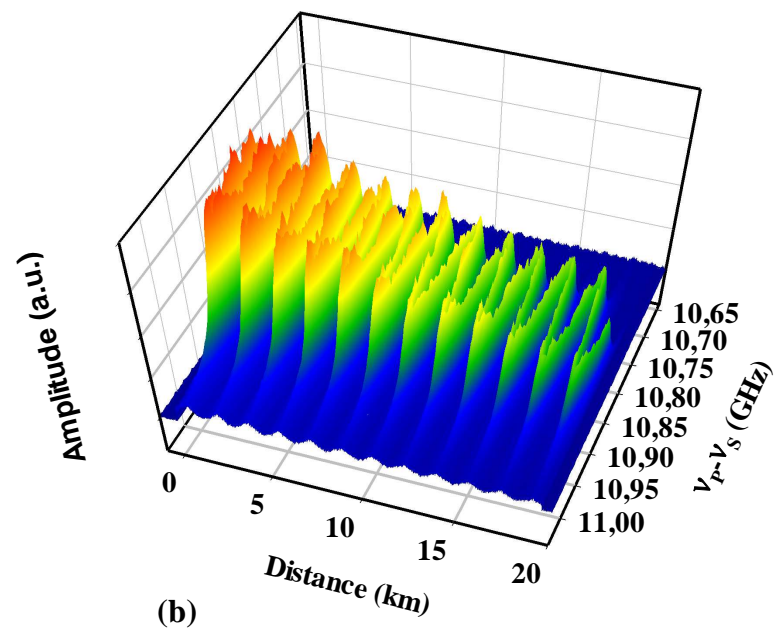

(b)

Figure 3. Experimental distribution of Brillouin spectra along the fiber for conventional BOTDA measurement (a) and (b) for a sinusoidal synthesized BFS distribution after applying modulation to the optical source.

Fig. 4 highlights the effects of BFS synthesis on the BOTDA sensor performance. Fig. 4(a) depicts how the depletion coefficient of the pump pulses is reduced when the virtual BFS is synthesized. The power of the injected probe wave was $0.34 \mathrm{~mW}$. Notice that application of the technique brings the coefficient below the $17 \%$ necessary to ensure less that $1^{\circ} \mathrm{C}$ measurement error in every circumstance ${ }^{1}$. On the other hand, in Fig. 4(b), the probability distribution of the detected 
BOTDA signal at a particular location is measured with and without optical source modulation to demonstrate the Brillouin-induced noise reduction feature. The standard deviation of noise was reduced by a factor of 1.42 . In addition, we also found that BFS synthesis increased the Brillouin threshold from aprox. 5-mW of probe power to values higher than those that could be obtained in our setup.

Finally, the BOTDA sensor with BFS synthesis was used for temperature measurements, obtaining for the particular experimental conditions used, $1-\mathrm{m}$ resolution and around $1{ }^{\circ} \mathrm{C}$ uncertainty at the end of the fiber (worst case).
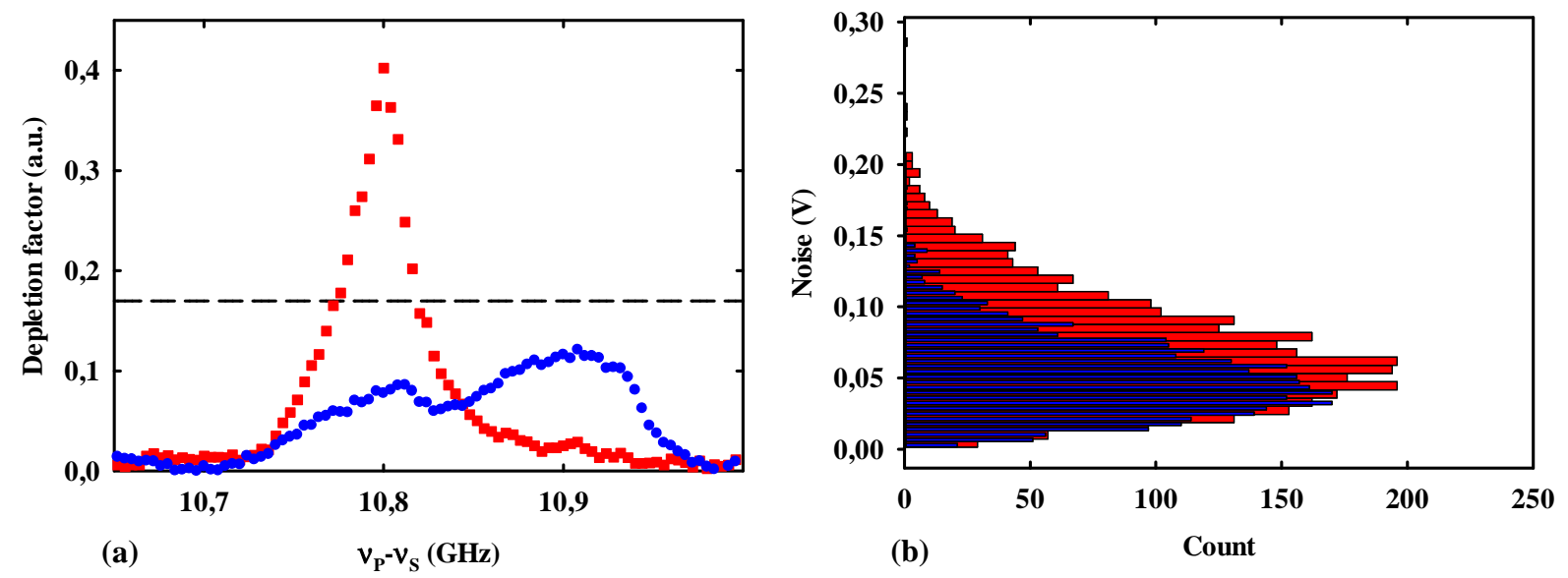

Figure 4. (a) Depletion coefficient of pump pulses as a function of the frequency separation between pump and probe with (blue circles) and without (red squares) source wavelength modulation and (b) detected noise histograms with (blue) and without (red) modulation.

\section{CONCLUSION}

In this work, we have presented a new concept for BOTDA sensors based on the synthesis of a virtual BFS profile along the sensing fiber. The technique relies on the wavelength modulation of an optical source using a periodic signal that is synchronized to the pulse generator. The presented experimental proof-of-concept has demonstrated the generation of this virtual BFS profile and its application to solving two of the fundamental problems presented in long-range BOTDA sensors: non-local effects and Brillouin threshold. However, this novel and simple tool is bound to find other interesting applications in Brillouin distributed sensing and signal processing.

\section{ACKNOWLEDGMENTS}

The authors wish to acknowledge the financial support from the Spanish Ministerio de Ciencia e Innovación through the project TEC2010-20224-C02-01 and from the Universidad Pública de Navarra.

\section{REFERENCES}

[1] Thévenaz, L., Mafang, S. and Lin, J., "Effect of pulse depletion in a Brillouin optical time-domain analysis system," Opt. Express 21(12), 14017-14035 (2013).

[2] Urricelqui, J., Sagues, M. and Loayssa, A., "BOTDA measurements tolerant to non-local effects by using a phase-modulated probe wave and RF demodulation," Opt. Express 21(14), 17186-17194 (2013).

[3] David, A. and Horowitz, M., "Low-frequency transmitted intensity noise induced by stimulated Brillouin scattering in optical fibers," Opt. Express 19(12), 11792-11803 (2011).

[4] Dong, Y., Chen, L. and Bao, X., "Extending the Sensing Range of Brillouin Optical Time-Domain Analysis Combining Frequency-Division Multiplexing and In-Line EDFAs," J. Lightwave Technol. 30(8), 1161-1167 (2012).

[5] Shiraki, K., Ohashi, M. and Tateda, M., "SBS threshold of a fiber with a Brillouin frequency shift distribution," J. Lightwave Technol. 14(1), 50-57 (1996). 
Copyright 2014 Society of Photo Optical Instrumentation Engineers. One print or electronic copy may be made for personal use only. Systematic re-production and distribution, duplication of any material in this paper for a fee or for commercial purposes, or modification of the content of the paper are prohibited. 